\title{
Gemeinsame Jahrestagung der Norddeutschen Röntgengesellschaft e. V. und der Röntgengesellschaft von Niedersachsen, Bremen und Sachsen-Anhalt e. V., 16./17.02.2018 in Hamburg
}

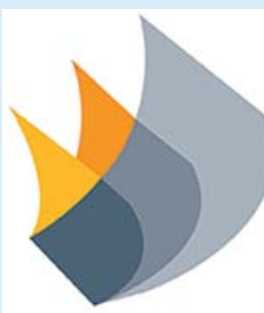

\section{RADIOLOGIEKONGRESSNORD2018} DER FORTBILDUNGSKONGRESS IM NORDEN

Liebe Kolleginnen und Kollegen,

schon 71 Jahre alt und seit 42 Jahren ein Verein. Das sind Eckdaten der Norddeutschen Röntgengesellschaft.

Damals wie heute teilten und teilen die Mitglieder der NDRG, Ärzte wie MTRA, die Begeisterung für die Radiologie in ihrer Breite und Tiefe in Bildgebung und interventioneller Therapie.

Und das wird auch zukünftig so sein.

Machen Sie sich selbst ein Bild davon, wie lebendig, wichtig, innovativ und zukunftsträchtig die Radiologie ist.

Ich lade Sie hiermit ganz herzlich zu dem Radiologiekongress Nord 2018 für Ärzte und MTRA am Freitag, den 16. und Samstag, den 17. Februar 2018 nach Hamburg ein. Wie die Jahre zuvor auch, ein Kongress in partnerschaftlicher Kooperation mit der Röntgengesellschaft von Niedersachsen, Bremen und Sachsen-Anhalt.

Freuen Sie sich auf lernreiche und interessante Tage mit den Themenschwerpunkten: Muskulo-Skelettal, Interventionen, Abdomen und Urogenital.

Es erwarten Sie anregende Plenumvorträge und lebhafte Fallbesprechungen in kleinen Gruppen mit spannenden Bildern zum Mitmachen.

Besonders am Herzen liegt mir, Sie auf den Q1- und Q2-Kurs für Kardio-CT aufmerksam zu machen.

Und zum Selbermachen bieten wir Ihnen ein Simulatorentraining, wo Sie Angiogra- fietechniken, inklusive gefäßverschließender und -eröffnender Verfahren ausprobieren und üben können.

Und so wie es im Berufsalltag eine enge und gute Zusammenarbeit zwischen Radiologen und MTRA gibt, wird auch dieser Kongress in Hamburg zusammen mit MTRA und in Kooperation mit der VMTB (Vereinigung Medizinisch-Technischer Berufe in der DRG) stattfinden, mit Vorträgen und Workshops speziell auf die Anforderungen der MTRA fokussiert.

Ich freue mich, Sie in Hamburg im Radisson Blue Hotel am Dammtor begrüßen zu dürfen. Nutzen Sie die Gelegenheit, mit namhaften Referenten, Vertretern der Industrie und anderen Teilnehmern ins Gespräch zu kommen; auf dem Kongress, oder in der Abendveranstaltung mit Musik und Kulinarischem.

\section{Schwerpunkte des Radiologiekongresses Nord 2018}

\section{Interventionelle Radiologie}

- Notwendige Diagnostik vor EVAR - Worauf muss ich achten

- EVAR - Materialien und Durchführung der Implantation

- Endoleak - Diagnostik und Therapie

\section{Abdomen}

- Fokale und diffuse Erkrankungen der Leber

- Onkologische, interventionelle Therapien bei Malignomen in der Leber

- TIPPS - gewusst wann und gewusst wie

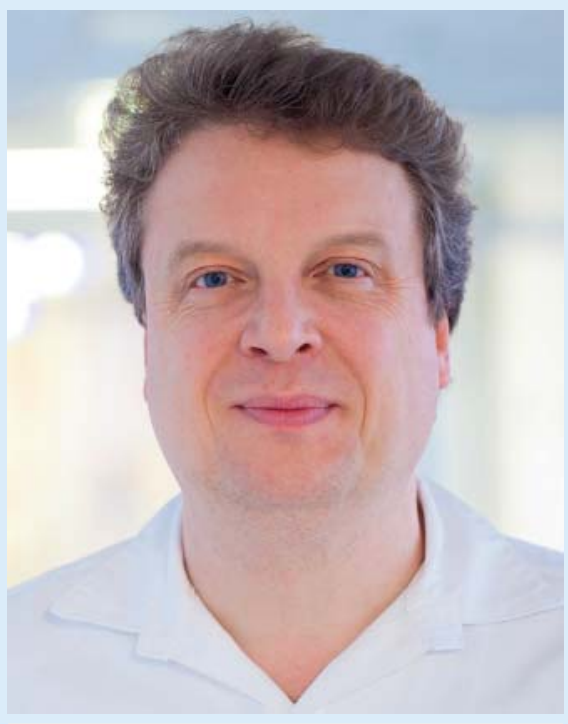

PD Dr. med. habil. Roger Eibel, Kongresspräsident Radiologiekongress Nord 2018, Helios Kliniken Schwerin

\section{Urogenital}

- Erkrankungen der Prostata

- Prostata-MRT - Durchführung und Befundung

- Nierenerkrankungen: Zysten, Karzinom und mehr

Muskulo-Skelettal mit den großen 6: Schulter, Ellenbogen und Handgelenk, Hüfte, Knie und Sprunggelenk

- Anatomie als Basis der Befundung

- Typische Erkrankungen aus Traumatologie, Orthopädie und Rheumatologie

- Was will der Zuweiser wissen?

Workshop für Ärzte : Q1- und Q2-KardioCT-Kurs

Workshop für MTRA in Kooperation mit dem Universitätsklinikum Eppendorf

Simulatortraining: Angiografieren und Gefäßinterventionen am Simulator für Ärzte und MTRA

Alle Informationen zum Kongress und die Möglichkeit zur Anmeldung finden Sie auf www.roeko-nord.de Bis 31.10.17 profitieren Sie vom Frühbuchertarif! 